\title{
El Servicio Europeo de Acción Exterior: un reto comunicativo para la diplomacia pública de la Unión Europea
}

\author{
Steffen Bay Rasmussen \\ Departamento de Relaciones Internacionales y Derecho Internacional Público \\ Facultad de Ciencias Sociales y de la Comunicación \\ Universidad del País Vasco / Euskal Herriko Unibertsitatea
}

\begin{abstract}
Sumario: I. Introducción.-II. La diplomacia pública de la Unión Europea: mensajes y prácticas principales. 1. Los mensajes: Proyección de identidad y difusión de valores. 2. Las prácticas de la diplomacia pública de la UE.-III. Las innovaciones del Tratado de Lisboa y el establecimiento del SEAE: continuidad y cambio potencial en la diplomacia pública.-IV. La creciente tensión en la diplomacia pública de la Unión: el impacto del establecimiento del SEAE. - V. Conclusiones.
\end{abstract}

Resumen: Este artículo analiza la diplomacia pública de la Unión Europea en el contexto de la entrada en vigor del Tratado de Lisboa y el establecimiento del nuevo Servicio Europeo de Acción Exterior (SEAE). Los mensajes de la diplomacia pública de la UE se estudian en términos de la proyección de su identidad y difusión de sus valores, así como la organización y prácticas de la comunicación externa de UE. El primer punto del estudio es sobre la continuidad y cambio potencial de la diplomacia pública de la UE, puesto que los cambios previstos con la institucionalización del SEAE todavía no se han materializado con respecto a la diplomacia pública. El segundo punto del estudio es sobre el reto a la diplomacia pública de la UE para transmitir su mensaje central de que la UE es un actor normativo cualitativamente diferente y un modelo para la paz estructural, cuando, de hecho, el SEAE implica un movimiento hacia una defensa cada vez más eficaz de los intereses materiales de la UE y visión euro-nacionalista de la UE como actor internacional.

Palabras clave: Unión Europea, diplomacia pública, Tratado de Lisboa, Servicio Europeo de Acción Exterior.

Abstract: This article analyses the EU's public diplomacy in the context of entry into force of the Lisbon Treaty and the establishment of the new European External Action Service (EEAS). It focuses on the messages of EU public diplomacy in terms of identity projection and value diffusion, as well as on the organization and practices of EU external communication. A first main argument is about the continuity and potential change of EU public diplomacy, as the changes foreseen with 
the institutionalisation of the EEAS have not yet materialised. A second main argument is the challenge to EU public diplomacy to convey its core message of the EU as being a qualitatively different normative actor and model for structural peace, when the EEAS in fact implies a movement towards an increasingly efficient defence of EU material interests and a euro-nationalist vision of the EU as an international actor.

Key words: European Union, public diplomacy, Lisbon Treaty, European External Action Service.

\section{Introducción}

La diplomacia de la Unión Europea ha estado evolucionando continuamente desde sus inicios en los años 50 y con el establecimiento del Servicio Europeo de Acción Exterior la Unión Europea ha entrado en una fase cualitativamente distinta de actuación internacional. Con el fin de contribuir al propósito general de esta edición especial de entender esta nueva fase de la evolución de la diplomacia de la UE, el presente artículo analiza el impacto de la reconfiguración de la Unión Europea como actor internacional en su diplomacia pública.

Aunque la diplomacia pública no es un fenómeno nuevo, con la intensificación de la globalización después del fin de la Guerra Fría, esta modalidad de diplomacia es cada vez más importante en relación a otras prácticas diplomáticas, un hecho reflejado tanto en las actividades y prioridades de los Ministerios de Asuntos Exteriores y en la atención académica generosa que ha recibido el fenómeno en la última década. La diplomacia pública es una modalidad de diplomacia que busca influencia política indirecta y estructural a través de influir en los discursos políticos dominantes en otras entidades políticas, por lo que son prácticas complementarias a las prácticas diplomáticas «tradicionales» que van dirigidas hacia gobiernos extranjeros ${ }^{1}$.

Debido a sus objetivos estratégicos, la diplomacia pública se convierte en una modalidad de actuación internacional particularmente importante para la Unión Europea. Sus objetivos estratégicos principales son la transformación estructural de terceros Estados y del sistema inter-

1 Son numerosas las definiciones de diplomacia pública. Para un análisis de éstas y un desarrollo más nítido de la presente definición, vid. RASMUSSEN, S.B., «The messages and practices of the European Union's public diplomacy» en Hague Journal of Diplomacy n. ${ }^{\circ} 2$, 2010, pp. 263-287. 
nacional ${ }^{2}$, por lo que, a través de lo que denomina Aldecoa una «política de responsabilidad $»^{3}$ y Keukeleire «diplomacia estructural» ${ }^{4}$, busca difundir los principios y valores europeos ${ }^{5}$, es decir, los principios de democracia, el Estado de derecho, el buen gobierno y los derechos humanos además de los valores de diálogo, búsqueda de consenso y la construcción de la interdependencia, multilateralismo e institucionalismo como formas de superar dilemas de seguridad en el sistema internacional westfaliano ${ }^{6}$. En este contexto, la diplomacia pública es una modalidad de diplomacia particularmente importante para la Unión Europea, puesto que la interacción diplomática tradicional entre representantes oficiales tiene el efecto principal de influir en el comportamiento inmediato de terceros Estados a través del uso de instrumentos como la condicionalidad económica. Para tener un impacto más profundo y a largo plazo es necesario, para la UE, que los principios y valores europeos se extiendan en las sociedades extranjeras, por lo que su comunicación directa con ellas asume una importancia especial.

El análisis del impacto del establecimiento del SEAE en la diplomacia pública de la Unión Europea está estructurado en tres partes principales. En la primera se examinarán los mensajes principales de la UE a las poblaciones de terceros Estados y las prácticas mediante las que la UE busca difundir sus mensajes. En la segunda parte, se detallarán las innovaciones principales de relevancia para la diplomacia pública del Tratado de Lisboa y el SEAE. La tercera parte consiste en un análisis de diferentes áreas de tensión en la diplomacia pública de la UE que constituirán los retos más importantes en los años que vienen.

${ }^{2}$ La Estrategia Europea de Seguridad definen esta doble transformación como necesaria para la seguridad de la UE. Vid. CONSEJO EUROPEO, Una Europa segura en un mundo mejor: Estrategia europea de seguridad, Bruselas, 2003.

3 ALDECOA LUZÁRRAGA, F., «La nueva política de responsabilidad y su dimensión ética» en Eikasia, n. ${ }^{\circ}$ 6, 2006; ALDECOA LUZẢRRAGA, F., «La política exterior común en el Tratado de Lisboa: instrumentos para hacer de la Unión un actor de la globalización» en FERNÁNDEZ LIESA, C.R. y DÍAZ BARRADO, C.M. (eds.), El Tratado de Lisboa: análisis y perspectivas, Dykinson, Madrid, 2008, pp, 234-251, pp. 233-235; ALDECOA LUZÁRRAGA, F. y GUINEA LLORENTE, M., La Europa que viene: el Tratado de Lisboa, Marcial Pons, Madrid, 2008, pp. 209-213.

${ }^{4}$ KEUKELEIRE, S., «The European Union as a diplomatic actor: Internal, structural, and traditional diplomacy» en Diplomacy and Statecraft, n. ${ }^{\circ} 3$, 2003, pp. 31-56; KEUKELEIRE, S., THIERS, U. y JUSTAERT, A., «Reappraising diplomacy: Structural diplomacy and the case of the European Union» en Hague Journal of Diplomacy, n. ${ }^{\circ}$ 2, 2009 , pp. 143-165.

${ }^{5}$ Formulados en el TRATADO DE LISBOA, artículo 21.

${ }^{6} \mathrm{La}$ promoción de estos mismos principios y valores constituyen el fundamento de la estrategia de comunicación externa de la UE, vid. COMISIÓN EUROPEA, The EU in the world: towards a communication policy for the European Union's external policy, 2006-2009, C (2006) 329/2 (Documento Interno de Trabajo), 2006. 


\section{La diplomacia pública de la Unión: mensajes y prácticas principales}

\section{Los mensajes: Proyección de identidad y difusión de valores}

Mientras es una verdad indiscutible que la UE no habla con una única voz, este hecho no conlleva necesariamente a que no exista, en un nivel abstracto, una auto-imagen y mensajes comunes que se transmiten por la cacofonía de voces de la UE. Diversidad es la marca de la Unión Europea, y esta marca no sólo se comunica a través de las diversas prácticas comunicativas, sino también se vive diariamente en el actual funcionamiento de la UE como actor. La base de esta concepción básica es la construcción políticamente poco controvertida de la UE como una entidad política, internamente diversa y compuesta por diferentes Estados que cooperan eficazmente para el bien común. Esta construcción básica conduce a la comunicación de dos conglomeraciones de mensajes.

La primera conglomeración construye la UE como un modelo para la paz y es la narrativa básica que ha acompañado la integración europea desde sus comienzos en los años 50. La «UE como un proyecto de paz» sigue siendo la identidad que busca comunicar la UE al mundo ${ }^{7}$. Mientras la UE se presenta al mundo como un proyecto de paz, se añade la idea de que también constituye un modelo que otros Estados y regiones del mundo deberían seguir ${ }^{8}$, con el argumento de que la experiencia europea de integración ha mostrado claramente el éxito del modelo. Parte del valor intrínseco de los principios fundadores de la UE, tales como estabilidad, democracia, derechos humanos, multilateralismo e igualdad entre Estados Miembros y solidaridad internacional ${ }^{9}$, y se considera que, debido a la universalidad de estos principios, deben adoptarse en el mundo en general, puesto que traen beneficios a todas las partes cuando usados como base de la interacción internacional y, más específicamente, en las instituciones de integración re-

7 Carta concluye sobre la base de 48 entrevistas con altos cargos de la Comisión sirviendo en las Delegaciones en el exterior que «la UE como modelo de paz» es la principal imagen que se comunica. Vid. CARTA, C., «The Commission's diplomats and the EU international image» en LUCARELLI, S., The external image of the European Union, Garnet Working Paper 17/2007, 2007. A una conclusión similar llega MICHALSKI, A., «The EU as a soft power: The force of persuasion» en MELISSEN, J. (ed.), The new public diplomacy: Soft power in international relations, Palgrave Macmilllan, Houndsmills, 2005, pp. 124-144.

${ }^{8}$ Visto por ejemplo en las campañas de diplomacia pública acompañando el 50 cumpleaños de la UE. Vid.: COMISIÓN EUROPEA, The EUs 50th anniversary celebrations around the world. A glance at EU public diplomacy at work, Brussels, 2007, pp. 5 y 11.

${ }^{9}$ Son los principios abstractos que define la UE en la estrategia de comunicación acompañando la política externa para describirse al mundo, vid.: COMISIÓN EUROPEA, op. cit., nota 6 . 
gional. Debido a esto, y a su naturaleza políticamente poca controvertida, los mensajes de democracia y derechos humanos son particularmente importantes para la UE en su diplomacia pública.

La segunda conglomeración de mensajes presenta a la UE como un actor internacional eficaz y se comunican los mensajes simultáneamente a audiencias internas y externas a la Unión Europea. La mayoría de los mensajes definidos explícitamente en la estrategia de comunicación de la política externa están así destinados tanto a legitimar que existe esta política de la UE, como a justificar su contenido específico. Otros mensajes comunican que la UE es un actor eficaz que consigue resultados por el dinero que gasta ${ }^{10}$. Así, un primer grupo de mensajes responde a la pregunta de por qué la UE tiene una política exterior, cuando los Estados Miembros ya llevan a cabo tantas actividades similares, lo que se hace a través de mensajes que sostienen que las actividades de la UE constituyen un valor añadido ${ }^{11}$ a las de los Estados Miembros. Un segundo grupo de mensajes responden a la pregunta de que en qué consiste la política exterior de la Unión. El argumento general en los mensajes es que la UE es un actor eficaz a la hora de promover estabilidad y prosperidad en el mundo y de que produce resultados concretos con respecto al desarrollo sostenible y la defensa global de los derechos humanos.

\section{Las prácticas de la diplomacia pública de la $U E^{12}$}

El enfoque general de la UE para influir en los discursos extranjeros es delegar las tareas a los órganos relevantes, con la coordinación central tratando sólo el nivel estratégico, sin entrar en detalles. Así, la UE nunca ha intentado hablar con una única voz en un sentido estricto, sino ha intentado, mediante una serie de mecanismos, coordinar las actividades de los diferentes actores de la red que constituye la UE como actor internacional para

${ }^{10}$ El eficaz uso de dinero era un tema al que volvieron los funcionarios de la Comisión en entrevistas semi-estructuradas llevadas a cabo por el autor, lo que sugiere una preocupación importante con este tema más allá de lo mencionado en la estrategia de comunicación. Las entrevistas se hicieron en mayo de 2008 tanto en la DG RELEX como en la DG Desarrollo y en EuropeAid por lo que se puede concluir que la preocupación con el gasto eficaz de dinero y la habilidad de la UE de producir resultados concretos es generalizada en la Comisión. Por supuesto, la preocupación no implica que la UE lo consiga, sino más bien que ha estado haciendo todo lo contrario e intentando mejorar.

${ }^{11}$ Como era el caso con el uso eficaz de dinero, el término «valor añadido» en referencia a las actividades de la Unión relativo a las de los Estados Miembros también era una expresión que usaron los entrevistados de la Comisión muy frecuentemente.

12 Dado el estado de desarrollo del SEAE en el momento de escribir estas líneas (noviembre de 2010), el análisis trata las prácticas antes del pleno funcionamiento del SEAE. 
que haya una cierta dirección común en las declaraciones, con el fin declarado de incrementar la visibilidad e influencia de la UE, y presentarse ante el mundo como un actor internacional coherente.

La coordinación más importante al nivel estratégico está llevada a cabo entre el Secretariado General del Consejo, el Alto Representante y la DG RELEX de la Comisión en el área de la PESC ${ }^{13}$. Las líneas políticas generales de comunicación en los diferentes ámbitos políticos se establecen en los relevantes grupos de trabajo del Consejo, que pueden pedir a las Delegaciones en el exterior que contribuyan con contenido y estrategias para la política de comunicación en cada ámbito ${ }^{14}$. En la definición final de la comunicación acompañando las diferentes políticas, varios funcionarios especialistas en la materia tanto del Secretariado General del Consejo como de la Comisión trabajan juntos.

Dentro de la Comisión también existe una gran necesidad de coordinar las varias DGs con competencias en política exterior, en su conjunto conocidas como la Familia RELEX. La coordinación se realiza, hasta la puesta en marcha del SEAE, en el Comité de Información Relex ${ }^{15}$, que se reúne cada mes. La idea es que las diferentes DGs de la Comisión con sus perspectivas diferentes de las relaciones externas de la Unión coordinen su comunicación externa, para que los mensajes específicos de cada DG resuenen con los mensajes generales que desea comunicar la UE. La intención no es que todas las DGs comuniquen los mismos mensajes, al contrario, cada DG es responsable de la comunicación externa dentro de su ámbito competencial y envía los mensajes específicos pertenecientes a su trabajo. La coordinación sólo busca evitar contradicciones y, cuando es posible, aprovechar sinergias al iniciar campañas coordinadas donde cada DG en su comunicación intenta poner el énfasis en un tema concreto.

Las Delegaciones en el exterior son los actores centrales en la diplomacia pública de la UE. El trabajo concreto y técnico de la diplomacia pública se hace en las Delegaciones, mientras los funcionarios en Bruselas se ocupan de la planificación estratégica de la comunicación y de coordinar el trabajo de las Delegaciones cuando las iniciativas de la diplomacia pública cubren más de un tercer Estado. La centralidad de las Delegaciones en la diplomacia pública se refleja en el presupuesto. Del presupuesto anual para la comunicación externa de 8 millones de euros, entre dos tercios y tres cuartos se destina al trabajo de las Delegaciones ${ }^{16}$.

${ }^{13}$ MICHALSKI, A., op. cit., nota 7, p. 132.

14 Entrevista en la DG RELEX de la Comisión, mayo de 2008.

15 Constituido por las DGs RELEX, Desarrollo, Comercio, Ampliación, AIDCO (EuropeAid), ECHO, ECFIN y PRESS.

16 Entrevista en la DG RELEX. 
Como consecuencia de la distribución desigual de fondos y personal, y también de la naturaleza desigual de las relaciones que tiene la Unión Europea con los terceros Estados, hay una gran diferencia entre las actividades de diplomacia pública que las Delegaciones son capaces de llevar a cabo, y, en consecuencia, es difícil pintar un cuadro general de las prácticas de la diplomacia pública de la Unión. Sin embargo, en un nivel abstracto, existe cierta similitud de enfoques. Las Delegaciones son responsables de identificar las audiencias destinatarias y llevar a cabo iniciativas específicas de comunicación ${ }^{17}$. Las actividades específicas dependen de la audiencia elegida, pero en general las Delegaciones concentran las iniciativas en «multiplicadores» como periodistas, ONGs y académicos, a través de la organización de conferencias, seminarios y visitas. Otra audiencia a la que, en general, se destina la comunicación es la gente joven ${ }^{18}$, pero dependiendo también del mensaje específico. Las actividades específicas incluyen, aparte de la organización de conferencias y seminarios, una amplia gama de actividades, desde la publicación de boletines de noticias, el mantenimiento de listas de correo, discursos y la gestión de páginas web, hasta la organización de ferias culinarias y competiciones deportivas.

Aparte de la comunicación oficial de Unión Europea dirigida hacia audiencias extranjeras, una forma complementaria de la diplomacia pública de la UE es la financiación de proyectos ejecutados por actores de la sociedad civil. Esta modalidad de diplomacia pública es particularmente relevante para afectar la definición e importancia de la democracia y los derechos humanos en discursos políticos extranjeros, por lo que se aplica principalmente hacia poblaciones de terceros Estados que la UE apoya a través de sus programas de ayuda al desarrollo ${ }^{19}$. El modus operandi básico de la UE es hacer a los actores de la sociedad civil solicitar financiación de la UE. Las propuestas de proyectos deben incluir, cuando no sean proyectos de comunicación, provisiones sobre la comunicación vinculada al proyecto, que especifican la audiencia, las formas de comunicar y los mensajes específicos ${ }^{20}$. En el proceso de elegir qué proyectos financiar, la evaluación de las propuestas recibidas incluye la comunicación, particularmente hasta qué punto el tema encuadra dentro de los mensajes generales de la UE y las

17 MICHALSKI, A., op. cit., nota 7, p. 133.

18 Identificado con un segmento de la sociedad de relevancia especial por la DG Desarrollo, vid.: COMISIÓN EUROPEA, DG Development information and communication strategy 2005-2009 and action plan for 2005, Bruselas, 2004.

${ }^{19}$ La UE también usa la modalidad en países industrializados, por ejemplo al financiar campañas de Greenpeace en Estados Unidos con el fin de influir en el discurso político sobre el cambio climático.

${ }^{20}$ COMISIÓN EUROPEA, Communication and visibility manual for EU external action, Bruselas, 2008. 
prioridades comunicativas, tal y como están definidos en la estrategia «La UE en el Mundo» ${ }^{21}$. El fin no es controlar los mensajes específicos sino asegurar la vinculación entre los proyectos financiados y los mensajes generales de la diplomacia pública de la Unión. ${ }^{22}$ El hecho de que no sea la Delegación la que los organice tiene la ventaja potencial de que los mensajes pueden ser más creíbles al provenir de una ONG supuestamente altruista, aunque esta ventaja se pierde, por lo menos en parte, por los requisitos de visibilidad que impone la UE sobre los proyectos que obligan a los socios a declarar el origen de sus fondos en todo el material publicado y otros sitios, reconociendo la financiación de la UE en los textos y debiendo exponer los símbolos de la $\mathrm{UE}^{23}$. El riesgo para la UE es que no se percibe como una entidad benévola con valores universales como quiere la UE, sino como un poder extranjero que quiere cambiar tradiciones locales e imponer valores nuevos y así promover sus propios intereses geopolíticos ${ }^{24}$.

\section{Las innovaciones del Tratado de Lisboa y el establecimiento del SEAE: continuidad y cambio potencial en la diplomacia pública}

El Tratado de Lisboa establece el SEAE como una de las novedades institucionales principales, aunque no establece nada sobre su funcionamiento u objetivos, aparte de apoyar a la Alta Representante de la Unión ${ }^{25}$. El fin específico del Tratado era generar más coherencia y continuidad en la acción y representación exterior de la UE al hacer a la Alta Representante responsable del conjunto de la política exterior. En este sentido, parece probable que la fusión de los puestos facilite el liderazgo e impulso político por la AP/VP, parcialmente en función de su derecho a presentar propuestas al Consejo ${ }^{26}$. Como Alta Representante será la representante de la Unión en materia de la PESC y responsable ante el Consejo, pero en otras materias actuará como Vicepresidenta de la Comisión y así será responsable ante el Colegio de Comisarios. De esta forma, la AR/VP será el nexo entre Consejo

${ }^{21}$ Entrevista en EuropeAid, mayo de 2008. La estrategia mencionada se detalla en los documentos: COMISIÓN EUROPEA, Europe in the world - some practical proposals for greater coherence, effectiveness and visibility, Bruselas, 2006; COMISIÓN EUROPEA, op. cit. nota 6.

22 Entrevista en EuropeAid, mayo de 2008.

${ }^{23}$ Las formas concretas se detallan en la publicación: Comisión Europea, op. cit. nota 19.

${ }^{24}$ Un dilema con respecto a visibilidad que también subraya SMITH, H., European Union foreign policy: What it is and what it does, Pluto Press, Londres, 2002, p. 95.

25 TRATADO DE LISBOA, artículo 27.

${ }^{26}$ CEBADA ROMERO, A., «Crónica de la acción exterior de la Unión Europea (enerojunio 2009)» en Revista Electrónica de Estudios Internacionales, n. ${ }^{\circ}$ 18, 2009, p. 2. 
y Comisión y, además, el nexo para la coordinación entre las varias DGs de la Comisión de la familia Relex y con respecto a las implicaciones exteriores de las políticas internas. El Tratado de Lisboa, por primera vez, crea una jerarquía entre Comisarios otorgando a la Vicepresidenta la autoridad de coordinar el trabajo de los demás Comisarios ${ }^{27}$. Su trabajo de coordinación con ambos órganos será vital para el éxito de las nuevas estructuras y el SEAE en particular, puesto que, como observan Edwards y Rijks, es más que probable que los problemas de competencia y luchas por poder entre las varias DGs de la Comisión, el Secretariado del Consejo y los diplomáticos de los Estados Miembros continuarán dentro de las nuevas estructuras y formas de interacción que conlleva la creación del SEAE ${ }^{28}$.

Aunque el SEAE es un órgano nuevo de la Unión Europea, su base ya existe en la forma de las Direcciones Generales de la Comisión y del Secretariado del Consejo que trabajan con asuntos exteriores. La administración central del SEAE se construirá importando los empleados y departamentos enteros relevantes del Secretariado del Consejo y de la Comisión. Desde el Secretariado General del Consejo se transfieren las unidades que trabajan en la PESC, es decir la Dirección General de Asuntos Exteriores y PolíticoMilitares y los órganos de la gestión diaria de la $\mathrm{PESC}^{29}$. De la Comisión se transfiere, en primer lugar, la DG RELEX. Como las demás Direcciones Generales con responsabilidades en el exterior, seguirá existiendo la DG Desarrollo como entidad diferenciada, aunque las Direcciones con responsabilidades geográficas de los Estados ACP se transfieren al SEAE. Así, en el SEAE habrá oficinas geográficas cubriendo todo el mundo. Además, el SEAE «contribuirá» a la programación y gestión de los instrumentos a la disposición de la DG Desarrollo, por ejemplo el Fondo Europeo de Desarrollo y el Instrumento Europeo para Democracia y Derechos Humanos. Más en concreto, el SEAE será «responsable de preparar las (...) decisiones de la Comisión» que tiene que ver con la elaboración de los documentos de estrategia regional y nacional ${ }^{30}$, es decir, de la programación plurianual y del trabajo geográficamente determinado de la DG Desarrollo. El establecimiento del SEAE entonces implica una reorganización importante de la Co-

27 TRATADO DE LISBOA, artículo 18.

28 CROWE, B., The European External Action Service. Roadmap for success, Royal Institute of International Affairs (Chatham House), Londres, 2008, p. 14; EDWARDS, G. y RIJKS, D., «Boundary problems in EU external representation» en INSTITUTO SUECO DE ESTUDIOS DE POLÍTICAS EUROPEAS (SIEPS) (ed.), Institutional competences in the EU external action: Actors and boundaries in CFSP and ESDP, SIEPS, Stockholm, 2008, pp. 15-95, pp. 73-75.

29 CONSEJO DE LA UNIÓN EUROPEA, Council decision establishing the organisation and functioning of the European External Action Service, Bruselas, 2010, Anexo.

${ }^{30}$ Ibid. 
misión con respecto a su actuación internacional hacia los países en vías de desarrollo.

Sobre el papel, el establecimiento del SEAE contiene el potencial de aumentar la coherencia de la actuación internacional de la UE en general, y también con respecto a la diplomacia pública en particular. Aunque la decisión del Consejo no contiene ningún detalle, está prevista la creación de un departamento de diplomacia pública en la administración central del SEAE. No existe todavía, y entonces es difícil estimar su impacto, aunque probablemente contribuirá a la profesionalización de la diplomacia pública y al tener un departamento dedicado, será más fácil coordinar el trabajo de las Delegaciones para obtener sinergias de su trabajo.

Sin embargo, en la formulación de los mensajes centrales de la diplomacia pública, el impacto probablemente no será muy grande. Los mensajes reflejan los valores de la PESC y los objetivos estratégicos de la Unión, sobre los que hay un amplio consenso en la Unión Europea. La diplomacia pública de la Unión consiste en la promoción de valores con los que es muy difícil no estar de acuerdo, y la identidad que busca proyectar la UE también está formulada en términos muy generales como un proyecto de paz y una diversidad que funciona. El SEAE contiene cambios con un impacto potencialmente mayor en la coordinación de la comunicación de las diferentes DGs de la Comisión que en la formulación de los mensajes. Como anteriormente era el Comisario Relex el responsable de coordinar los mensajes de la Comisión y coordinar con el Consejo, ahora es la Alta Representante, lo que sugiere cierta continuidad en la coordinación. Sin embargo, se ha establecido una clara jerarquía, lo que debería facilitar la coordinación en este nivel más abstracto. El departamento del SEAE dedicado a la diplomacia pública tendrá probablemente un papel clave en esta coordinación al nivel de iniciativas y campañas concretas, por lo que el nuevo departamento de diplomacia pública tendrá el potencial de aumentar la coordinación con respecto los mecanismos de planificación y coordinación existentes.

Con respecto a la ejecución de la diplomacia pública en el exterior, el cuadro general también es de cierta continuidad y de posibilidades de cambio. La base del funcionamiento del SEAE en el exterior serán las Delegaciones de la Comisión que se convertirán en Delegaciones de la Unión con el papel de representar a la Unión Europea en vez de sólo a la Comisión ${ }^{31}$. Que la Delegación de la UE asuma así el papel de la Presidencia rotatoria proveerá a la representación de la Unión de más continuidad y menos complejidad que la formula de representación múltiple de la UE por la misión diplomática de la Presidencia del Consejo y Delegación de la Comisión.

31 TRATADO DE LISBOA, artículo 221. 
Una posible complicación en el trabajo de las Delegaciones es que trabajarán simultáneamente para diferentes burocracias en Bruselas. Por una parte estará la burocracia propia del SEAE, fusionando las DGs del Secretariado del Consejo trabajando con la PESC y la DG RELEX de la Comisión, unificando así la representación de los pilares. El Jefe de Delegación será, entonces, parte del SEAE. Por otra parte, otras DGS de la Comisión mantendrán su administración aparte, por lo que las Delegaciones también trabajaran con asuntos que no forman parte de la cartera de la burocracia del SEAE en Bruselas, sino de otras DGs de la Comisión, como Desarrollo, Ampliación, Comercio o Medio Ambiente. Aún así, el Jefe de Delegación será responsable del conjunto del trabajo de la Delegación y de su coherencia ${ }^{32}$, lo que le otorga cierto poder con respecto al personal que no forma parte del SEAE. De esta forma, parece que los funcionarios en las Delegaciones de las varias DGs de la Comisión seguirán llevando a cabo sus iniciativas concretas vinculadas a proyectos de la sociedad civil, organizando iniciativas comunicativas específicas relativas a su área de trabajo y participando en el debate público en el tercer Estado donde trabajan. Aún así, las unidades de comunicación o diplomacia pública en las Delegaciones pasarán a formar parte del SEAE, por lo que también en el exterior el SEAE contendrá el potencial de aumentar la coherencia de la comunicación de la UE y de obtener sinergias de su trabajo, tanto a nivel estratégico para organizar la comunicación en determinados temas, como a nivel práctico para tomar iniciativas que incluyen la comunicación de los diferentes ámbitos de trabajo de las Delegaciones.

Aparte del potencial de una mejor coordinación en la comunicación externa de la UE, el hecho de tener un Servicio Exterior en funcionamiento y encabezado por una Alta Representante debe tener un efecto marcado en la visibilidad de la Unión Europea como actor internacional ${ }^{33}$. Es demasiado pronto para estimar el impacto resultante en la identidad de la UE como actor pero, sin duda, la diplomacia pública de la Unión tendrá a partir de ahora menos retos en el sentido de hacer visible la UE en el escenario internacional. Particularmente, la unificación representativa de los pilares en la Alta Representante y los Jefes de las Delegaciones contribuirán a la visibilidad de la UE. De tal forma, aunque los mensajes de la Unión probablemente no cambiarán mucho, quizá será más fácil para sus representantes influir en el debate público global y en terceros Estados, puesto que podrán hablar más libremente. Sin embargo, además de la tarea comunicativa de

32 CONSEJO DE LA UNIÓN EUROPEA, op. cit., nota 28, artículo 5.

33 GUTIÉRREZ ESPADA, C. y CERVELL HORTAL, M.J., La adaptación al tratado de Lisboa (2007) del sistema institucional decisorio de la Unión, su acción exterior y personalidad jurídica, Comares, Granada, 2010, p. 95. 
explicar el nuevo funcionamiento de la Unión, un aumento de la visibilidad de la Unión como actor también conlleva nuevo retos vinculados a la existencia de varias tensiones en la diplomacia de la Unión en general, y en su diplomacia pública en particular. Estas áreas de tensión son el objeto del análisis en la siguiente sección.

\section{La creciente tensión en la diplomacia pública de la Unión: el impacto del establecimiento del SEAE}

Una primera tensión identificable aparece entre la necesidad de coherencia y la necesidad de descentralizar la diplomacia pública para que tenga un impacto en los discursos políticos en terceros Estados. Tanto la literatura teórica sobre la diplomacia pública como las experiencias prácticas apuntan a la necesidad de adaptar la comunicación a cada audiencia, tanto en términos de mensajes como de prácticas: «Si queremos tener éxito en nuestros esfuerzos para que se entienda nuestra sociedad y nuestras políticas, primero debemos entender los motivos, cultura, historia y psicología de la gente con quien queremos comunicar ${ }^{34} \gg$. También es importante mantener un proceso de comunicación abierto, de entrar en un diálogo ${ }^{35}$, puesto que el mensaje debe ajustarse continuamente, dependiendo de su impacto en el discurso objetivo del mensaje ${ }^{36}$. Este hecho hace necesaria una gran flexibilidad a la hora de planificar y ejecutar iniciativas de diplomacia pública. En consecuencia, van Ham concluye que la experiencia del enfoque «talla única» de los EE.UU. a la diplomacia pública ha sido un fracaso ${ }^{37}$, y Noya concluye, en la misma línea, que los funcionarios que ejecutan la diplomacia pública deben ser especialistas en áreas o regiones específicas ${ }^{38}$. De ahí

${ }^{34}$ Gifford D. Malone, citado por POTTER, E.H., Canada and the new public diplomacy, Discussion Paper in Diplomacy n. ${ }^{\circ}$ 81, Netherlands Institute of International Relations «Clingendael», La Haya, 2002, p. 3.

35 MELISSEN, J., «The new public diplomacy: Between theory and practice» en MELISSEN, J. (ed.), The new public diplomacy: Soft power in international relations, Palgrave Macmilllan, Houndsmills, 2005, pp. 3-27, pp. 13-14.

${ }^{36}$ Leonard añade a este argumento que mensajes unidireccionales son propensos a crear resentimiento en públicos extranjeros. Vid.: LEONARD, M., STEAD, C. y SMEWING, C., Public diplomacy, The Foreign Policy Centre, Londres, 2002, p. 48.

37 VAN HAM, P., «Power, public diplomacy, and the Pax Americana» en MELISSEN, J. (ed.), The new public diplomacy: Soft power in international relations, Palgrave Macmilllan, Houndsmills, 2005, pp. 47-66, pp. 60-61. A conclusiones similares llega MELISSEN, J., «Public diplomacy between theory and practice» en NOYA, J. (ed.), The present and future of public diplomacy: a European Perspective. The 2006 Madrid conference on public diplomacy, Real Instituto Elcano, Madrid, 2006.

38 NOYA, J., Diplomacia pública para el siglo XXI, Ariel, Barcelona, 2007, p. 155. 
que la organización de la UE en una red de actores con responsabilidad fijada para cada uno dentro de su área es apta para una diplomacia pública eficaz. Sin embargo, esta organización también hace imposible hablar con una única voz. El reto es coordinativo, por lo que la Comisión ha estado llevando a cabo varias iniciativas para aumentar la coherencia entre diferentes mensajes y cosechar las sinergias entre diferentes prácticas específicas. En este sentido, es una tensión que probablemente disminuya en el futuro, si es que llega a funcionar como parte de las estructuras del SEAE un departamento de diplomacia pública coordinando la comunicación central de Bruselas y el trabajo comunicativo de las Delegaciones.

Una crítica de la diplomacia de la UE en general, y de su diplomacia pública en particular, es su falta de coherencia debido a la multitud de actores que la realiza, lo que ha hecho surgir llamamientos ${ }^{39}$ para una mejor planificación estratégica de la diplomacia pública y para cambios estructurales que permitan que la UE hable con una única voz ${ }^{40}$. El SEAE ha de interpretarse como la respuesta a estos llamamientos, particularmente el establecimiento del departamento de diplomacia pública. Sin embargo, hablar con una voz y aumentar la visibilidad de la Unión en las relaciones internacionales contiene un peligro, puesto que podría aumentar la aversión de las audiencias extranjeras hacia lo que, a veces, perciben como una imposición de principios perniciosos por parte de un poder extranjero que defiende sus intereses materiales. Este hecho dirige la atención a una segunda tensión detectable en la diplomacia pública de la Unión Europea que persistirá, e incluso puede aumentar, con el SEAE en marcha. Es la tensión entre la visibilidad de la UE como actor y la proyección de su identidad como actor fuerte, por un lado, y el objetivo de difundir los valores europeos, por otro lado. La proyección de la identidad de la UE requiere aumentar la visibilidad de la UE, pero esta visibilidad, al mismo tiempo, debilita la difusión normativa. El argumento es simple: si una ONG en terceros Estados pone banderas de la UE en sus comunicaciones y reconoce la financiación, se debilita su mensaje de derechos humanos o de la protección del medio ambiente, puesto que los mensajes así se construyen como perteneciendo a los intereses de la UE, una imposición externa, y no algo inherente en la sociedad del tercer Estado. Esta relación inversa entre las posibilidades de actuar coherentemente y proyectar una identidad como actor fuerte y conseguir los

39 FISKE DE GOUVEIA, Philip, European infopolitik: Developing EU public diplomacy strategy, The Foreign Policy Centre, Londres, 2005; LYNCH, D., Communicating Europe to the world: what public diplomacy for the EU, EPC Working Paper n. ${ }^{\circ} 21$, Bruselas, European Policy Centre, 2005.

${ }^{40}$ Hablar con una única voz es para Michalski una necesidad obvia para que la UE sea capaz de promover sus valores, vid.: MICHALSKI, A., op. cit., nota 7, p. 141. 
objetivos transformativos de la diplomacia pública, se debe, entonces, a que la UE se podrá percibir como un poder tradicional que defiende sus intereses a corto plazo en vez de como un nuevo actor benigno que se basa en valores e ideas universales.

La tensión entre visibilidad y difusión de valores mediante la diplomacia pública está vinculada a otra tensión detectable en la diplomacia pública de la Unión que también es probable que aumente con el SEAE en marcha. Se puede interpretar el SEAE como un intento de convertir la organización en red de la Unión Europea como actor diplomático en algo que parezca (un poco) más al ideal westfaliano de un actor unitario, para que la UE sea capaz de defender sus intereses materiales cortoplacistas mediante el empleo de las prácticas diplomáticas habituales del sistema westfaliano. Con respecto a la organización en Bruselas, se crea un nuevo órgano fuerte que debe aliviar los problemas de coherencia horizontal entre las DGs de la Comisión y entre la Comisión y el Consejo. En el exterior también, las nuevas Delegaciones de la Unión serán una presencia única y continua de la Unión Europea. Aunque varios analistas coinciden en que Lisboa y el SEAE contienen el potencial de mejorar la coherencia, no lo garantiza ${ }^{41}$, y se puede interpretar que lo que está intentando la UE con el Tratado de Lisboa y el establecimiento del SEAE es equiparse como actor para poder funcionar y defender sus intereses en un sistema internacional caracterizado por una cultura Lockeana de coexistencia competitiva, o incluso Hobbesiana de enemistad ${ }^{42}$.

Esto corresponde a la visión del mundo de Robert Cooper, recién nombrado alto cargo del SEAE, que opina que lo que debe hacer la UE es mantener su cultura Kantiana internamente y ser actor según las normas de la cultura Lockeana externamente. «Entre nosotros, mantengamos la ley, pero cuando actuamos en la selva, debemos usar las leyes de la selva ${ }^{43}$. Su visión, que sin duda es más realista a corto plazo, conlleva el peligro de que los objetivos de transformación estructural pierdan fuerza a largo plazo debido a la transformación de la propia Unión. Así, observa Martin Ortega, la UE no podrá existir y prosperar como entidad política en un sistema in-

${ }^{41}$ CROWE, B., op. cit., nota 27, p. 10; DUKE, S., «Providing for European-level diplomacy after Lisbon: The case of the European External Action Service» en Hague Journal of Diplomacy, n. ${ }^{\circ}$ 2, 2009, pp. 211-233, p. 213; FERNÁNDEZ SOLA, N., El Servicio de Acción Exterior de la Unión Europea, Documento de Trabajo n. ${ }^{\circ}$ 46/2008, Real Instituto Elcano, Madrid, 2008, pp. 6-7.

42 Para una explicación detallada de las culturas Hobbesiana, Lockeana y Kantiana de anarquía, vid. WENDT, A., Social theory of international politics, Cambridge University Press, Cambridge, 1999, pp. 246-313.

${ }^{43}$ COOPER, R., The post-modern state and the world order, Demos, Londres, 2000, p. 38 . 
ternacional Hobbesiano sino depende de la transformación del sistema internacional en una comunidad global de naturaleza Kantiana para poder prosperar ${ }^{44}$, lo que también es la idea causal principal en la Estrategia de Seguridad de la UE ${ }^{45}$. Usando el lenguaje de los paradigmas inconmensurables, Ortega argumentó en 2007 que la UE se encontraba en el «momento de la verdad» donde tenía que elegir entre el paradigma de intereses o el paradigma de principios ${ }^{46}$. Cabe notar que en los últimos años hemos visto una priorización de los intereses por encima de los principios, evidente sobre todo dentro del marco de la Unión por el Mediterráneo, donde los intereses de la UE de estabilidad política en los Estados vecinos y la cooperación en temas de migración ha caracterizada las relaciones, y la promoción de los valores de la UE de democracia y derechos humanos ha sido relegada a un segundo plano ${ }^{47}$. En este contexto, parece que la UE con la reorganización de su diplomacia apuesta por el discurso euro-nacionalista ${ }^{48}$ de la UE como un actor internacional que defiende sus intereses materiales a corto plazo de forma eficaz.

Existe una tensión creciente entre la diplomacia «tradicional» de la UE, que con el SEAE será en mayor medida una representación de un actor coherente y determinado, y la diplomacia pública que busca difundir una imagen de la UE como un proyecto de paz y una alternativa a las dinámicas de alienación entre los Estados en el sistema westfaliano. El dilema surge del hecho de que la UE pretende influir en el mundo de dos formas simultáneamente: como modelo a seguir y como actor fuerte ${ }^{49}$ y que, hasta cierto punto, las dos formas son incompatibles. En el vocabulario de Wendt, el argumento básico es que la Unión no puede contribuir a construir una comunidad internacional al nivel global caracterizada por una cultura Kantiana de anarquía, si se comporta como un actor Lockeano que busca defender sus intereses en un sistema de coexistencia competitiva entre actores soberanos westfalianos.

${ }^{44}$ ORTEGA, M., Building the future. The EU's contribution to global governance, Chaillot Paper n. ${ }^{\circ}$ 100, Instituto de Estudios de Seguridad de la Unión Europea (EUISS), 2007, p. 97.

45 Vid. CONSEJO EUROPEO, op. cit. nota 2.

46 ORTEGA, M., op. cit. nota 43, pp. 100-103.

47 Vid. BARBÉ IZUEL, E.,» La Unión por el Mediterráneo: de la europeización de la política exterior a la descomunitarización de la política mediterránea» en Revista de Derecho Comunitario Europeo, n. ${ }^{\circ} 32,2009$, pp. 11-48; YOUNGS, R., «Europa y la promoción de la democracia en el sur del Mediterráneo» en BARBÉ IZUEL, E. y HERRANZ, A. (eds.), Política exterior y Parlamento Europeo: hacia el equilibrio entre eficacia y democracia, Oficina del Parlamento Europeo en Barcelona, Barcelona, 2007.

${ }^{48}$ La expresión es de CAMERON, F., An introduction to European foreign policy, Nueva York, Routledge, 2007, p. 216.

49 ORTEGA, M., op. cit., nota 43, pp. 91-94. 
Este potencial conflicto entre diferentes objetivos es un problema general en la diplomacia de la Unión Europea y, en este sentido, el establecimiento del SEAE constituye un reto comunicativo importante para la diplomacia pública de la Unión Europea. Por un lado, el SEAE contribuye a crear dinámicas más fuertes de «nosotros» y «ellos,» entre la UE y otros Estados, mientras al mismo tiempo es objetivo de la UE debilitar, o incluso superar, estas mismas dinámicas mediante la exportación de su modelo de paz estructural. Mensajes relativos a la proyección de una identidad de la UE como actor fuerte capaz de hacer una diferencia real en el mundo serán más fáciles de comunicar. En contraste, será cada vez más difícil difundir el mensaje de la UE como un modelo de paz estructural y un actor altruista que sólo quiere difundir sus valores para que otras partes del mundo tengan las mismas experiencias positivas.

Una posible forma de impedir a medio y largo plazo que el establecimiento del SEAE tenga un impacto demasiado negativo en la comunicación del mensaje de la UE como una comunidad de valores y un modelo para la paz, y en sus objetivos estratégicos de transformación interna de otros Estados, es la promoción, más activamente, de la cultura europea. Permitiría dar contenido al lema de «unida en diversidad» y comunicar una identidad de la UE más esencialista y tangible, aunque sin fortalecer las dinámicas de «nosotros» y «ellos» en la diplomacia de la UE. Es dudoso si la cartera del futuro departamento de diplomacia pública en el SEAE incluirá también la diplomacia cultural, dada su ausencia en los documentos y discursos oficiales. Una forma, quizá mejor, de incorporar la dimensión cultural en la diplomacia pública de la UE es una cooperación estructurada con la red de los Institutos Nacionales de Cultura de la Unión Europea (EUNIC), puesto que estos institutos ya están presentes en muchos terceros Estados y cuentan con años de experiencia en la materia.

\section{Conclusiones}

Este artículo sobre el impacto del Servicio Europeo de Acción Exterior se ha enfocado en la diplomacia pública de la Unión Europea. El análisis permite llegar a varias conclusiones. En primer lugar, es importante resaltar la descentralización de la comunicación externa de la Unión Europea. Esto implica que los diferentes órganos de la Unión Europea son los responsables de la comunicación estratégica con poblaciones extranjeras dentro de su área de trabajo. Gran parte de esta comunicación la llevan a cabo las Delegaciones en el exterior. Así, la organización en una red de la diplomacia pública de la Unión Europea corresponde generalmente a las recomendaciones de la literatura teórica sobre diplomacia pública. 
La descentralización implica un reto de coordinación importante. En este sentido, destaca la creación en el SEAE de una sección dedicada a la diplomacia pública y cultural. Con respecto a las prácticas de la diplomacia pública de la Unión se puede categorizar el probable funcionamiento del SEAE como una mejora notable de los mecanismos existentes de coordinación, que además respeta la necesidad de la organización de la diplomacia pública de la Unión en una red.

Con respecto a los mensajes de la diplomacia pública, el SEAE crea un reto comunicativo importante. Aparte de la labor de explicar en qué consisten las nuevas estructuras de la Unión Europea, el desarrollo de la UE como actor internacional, representado por el SEAE, hace más difícil la comunicación del mensaje central de la diplomacia pública sobre la UE como un modelo para la paz estructural entre Estados y la viabilidad de la UE como «unida en diversidad». El incremento de la visibilidad de la UE como actor y de la capacidad de acción decisiva que conlleva el SEAE a la hora de defender los intereses materiales de la UE, hacen más difícil de comunicar la imagen de ésta como un actor benigno o normativo cualitativamente diferente de los Estados westfalianos. Este reto comunicativo es una expresión más de una tensión generalizada en la diplomacia de la Unión entre la utopía universalista que es el fundamento de la identidad internacional de la UE y su necesidad de defender sus intereses frente a terceros Estados en un sistema internacional todavía de corte westfaliano. Al no resolverse esta tensión mediante la clara apuesta por la exportación del modelo europeo de coexistencia pacífica entre Estados en vez de por la euro-nacionalización de la UE como actor internacional, seguirá aumentando el reto comunicativo que constituye el SEAE para la diplomacia pública. $\mathrm{Si}$, en cambio, la UE a medio plazo se convierte en un actor fuerte, capaz de defender sus intereses de forma eficaz con todos los medios diplomáticos y militares y, en general, sigue su evolución hacia priorizar de forma sistemática la defensa de sus intereses por encima de la difusión de sus valores, será necesaria una reformulación total de los mensajes de la diplomacia pública de la Unión.

\section{Bibliografía}

ALDECOA LUZÁRRAGA, F., «La nueva política de responsabilidad y su dimensión ética» en Eikasia, n. ${ }^{\circ}$ 6, 2006.

ALDECOA LUZÁRRAGA, F., «La política exterior común en el Tratado de Lisboa: instrumentos para hacer de la Unión un actor de la globalización» en FERNÁNDEZ LIESA, C.R. y DÍAZ BARRADO, C.M. (eds.), El Tratado de Lisboa: análisis y perspectivas, Dykinson, Madrid, 2008, pp, 234-251. 
ALDECOA LUZÁRRAGA, F. y GUINEA LLORENTE, M., La Europa que viene: el Tratado de Lisboa, Marcial Pons, Madrid, 2008, pp. 209-213.

BARBÉ IZUEL, E., «La Unión por el Mediterráneo: de la europeización de la política exterior a la descomunitarización de la política mediterránea» en Revista de Derecho Comunitario Europeo, n. ${ }^{\circ}$ 32, 2009.

CARTA, C., «The Commission's diplomats and the EU international image» en LUCARELLI, S., The external image of the European Union, Garnet Working Paper 17/2007, 2007.

CEBADA ROMERO, A., «Crónica de la acción exterior de la Unión Europea (enero-junio 2009)» en Revista Electrónica de Estudios Internacionales, n. ${ }^{\circ} 18$, 2009.

COMISIÓN EUROPEA, DG Development information and communication strategy 2005-2009 and action plan for 2005, Bruselas, 2004.

COMISIÓN EUROPEA, Europe in the world - some practical proposals for greater coherence, effectiveness and visibility, Bruselas, 2006.

COMISIÓN EUROPEA, The EU in the world: towards a communication policy for the European Union's external policy, 2006-2009, C (2006) 329/2 (Documento Interno de Trabajo), 2006.

COMISIÓN EUROPEA, The EUs 50th anniversary celebrations around the world. A glance at EU public diplomacy at work, Brussels, 2007.

COMISIÓN EUROPEA, Communication and visibility manual for EU external action, Bruselas, 2008.

CONSEJO EUROPEO, Una Europa segura en un mundo mejor: Estrategia europea de seguridad, Bruselas, 2003.

CONSEJO DE LA UNIÓN EUROPEA, Council decision establishing the organisation and functioning of the European External Action Service, Bruselas, 2010.

COOPER, R., The post-modern state and the world order, Demos, Londres, 2000.

CROWE, B., The European External Action Service. Roadmap for success, Royal Institute of International Affairs (Chatham House), Londres, 2008.

DUKE, S., «Providing for European-level diplomacy after Lisbon: The case of the European External Action Service» en Hague Journal of Diplomacy, n. ${ }^{\circ} 2$, 2009, pp. 211-233.

EDWARDS, G. y RIJKS, D., «Boundary problems in EU external representation» en INSTITUTO SUECO DE ESTUDIOS DE POLÍTICAS EUROPEAS (SIEPS) (ed.), Institutional competences in the EU external action: Actors and boundaries in CFSP and ESDP, SIEPS, Stockholm, 2008, pp. 15-95

FERNÁNDEZ SOLA, N., El Servicio de Acción Exterior de la Unión Europea, Documento de Trabajo n. ${ }^{\circ}$ 46/2008, Real Instituto Elcano, Madrid, 2008.

FISKE DE GOUVEIA, Philip, European infopolitik: Developing EU public diplomacy strategy, The Foreign Policy Centre, Londres, 2005.

GUTIÉRREZ ESPADA, C. y CERVELL HORTAL, M.J., La adaptación al tratado de Lisboa (2007) del sistema institucional decisorio de la Unión, su acción exterior y personalidad jurídica, Comares, Granada, 2010

KEUKELEIRE, S., «The European Union as a diplomatic actor: Internal, structural, and traditional diplomacy» en Diplomacy and Statecraft, n. ${ }^{\circ} 3,2003$, pp. 31-56. 
KEUKELEIRE, S., THIERS, U. y JUSTAERT, A., «Reappraising diplomacy: Structural diplomacy and the case of the European Union» en Hague Journal of Diplomacy, n. ${ }^{\circ} 2,2009$, pp. 143-165.

LEONARD, M., STEAD, C. y SMEWING, C., Public diplomacy, The Foreign Policy Centre, Londres, 2002.

LYNCH, D., Communicating Europe to the world: what public diplomacy for the $E U$, EPC Working Paper n. ${ }^{\circ}$ 21, Bruselas, European Policy Centre, 2005.

MELISSEN, J., «The new public diplomacy: Between theory and practice» en MELISSEN, J. (ed.), The new public diplomacy: Soft power in international relations, Palgrave Macmilllan, Houndsmills, 2005, pp. 3-27.

MELISSEN, J., «Public diplomacy between theory and practice» en NOYA, J. (ed.), The present and future of public diplomacy: a European Perspective. The 2006 Madrid conference on public diplomacy, Real Instituto Elcano, Madrid, 2006.

MICHALSKI, A., «The EU as a soft power: The force of persuasion», en MELISSEN, J. (ed.), The new public diplomacy: Soft power in international relations, Palgrave Macmilllan, Houndsmills, 2005, pp. 124-144.

NOYA, J., Diplomacia pública para el siglo XXI, Ariel, Barcelona, 2007.

ORTEGA, M., Building the future. The EU's contribution to global governance, Chaillot Paper n. ${ }^{\circ}$ 100, Instituto de Estudios de Seguridad de la Unión Europea (EUISS), 2007.

POTTER, E. H., Canada and the new public diplomacy, Discussion Paper in Diplomacy n. $^{\circ} 81$, Netherlands Institute of International Relations «Clingendael», La Haya, 2002.

RASMUSSEN, S. B., «The messages and practices of the European Union's public diplomacy» en Hague Journal of Diplomacy n. ${ }^{\circ}$ 2, 2010, pp. 263-287.

SMITH, H., European Union foreign policy: What it is and what it does, Pluto Press, Londres, 2002.

VAN HAM, P., «Power, public diplomacy, and the Pax Americana» en MELISSEN, J. (ed.), The new public diplomacy: Soft power in international relations, Palgrave Macmilllan, Houndsmills, 2005, pp. 47-66.

WENDT, A., Social theory of international politics, Cambridge University Press, Cambridge, 1999

YOUNGS, R., «Europa y la promoción de la democracia en el sur del Mediterráneo» en BARBÉ IZUEL, E. y HERRANZ, A. (eds.), Política exterior y Parlamento Europeo: hacia el equilibrio entre eficacia y democracia, Oficina del Parlamento Europeo en Barcelona, Barcelona, 2007. 